Communications in Physics, Vol. 23, No. 2 (2013), pp. 121-125

\title{
STUDY ON THE PHASE TRANSITION BEHAVIOR OF FISHES SCHOOLING SYSTEM
}

\author{
NGUYEN PHUOC THE \\ Department of Natural Science, Duy Tan University, \\ Kr/25 Quang Trung, Hai Chau, Da Nang, Vietnam \\ LEE SANG-HEE \\ Division of Fusion and Convergence of Mathematical Sciences, \\ National Institute for Mathematical Sciences, Daejeon, Republic of Korea \\ NGO VAN THANH AND NGUYEN AI VIET \\ Institute of Physics, \\ Vietnam Academy of Science and Technology \\ 10 Dao Tan, Ba Dinh, Hanoi, Vietnam \\ Email: nvthanh@iop.vast.ac.vn \\ Received 25 May 2013 \\ Accepted for publication 08 June 2013
}

\begin{abstract}
The Vicsek's model (VM) [T. Vicsek, et. al., Phys. Rev. Lett. 75 (1995) 1226] is a popular approach to study nature of the phase transition of self-propelling system. In this model, the direction of motion of each particle depends on the average velocity of its neighboring particles within a circle of radius $R$. In this work, we consider a group of fishes as the particles, but we assumed that only the individuals of its neighbors within a circle sector with central angle $\phi$ (is called angle of view) and radius $R$, are taken into account the average direction of motion. We used a similar model as the well known XY spin model on a square lattice of linear size $L$, the particles can move freely on the plane (an off-lattice model). A phase diagram in the space ( $\varphi$, $\left.\xi_{c}\right)$ has been established where $\varphi=\phi / 2 \in[0, \pi]$ and $\xi_{c}$ being the critical noise. We showed that $\xi_{c}$ strongly depends on the view's angle $\varphi \geq 0.5 \pi$, but slightly varies with $\varphi<0.5 \pi$. So, we can conclude that the schooling behavior of animal is only of the prey species, but not of the predator.
\end{abstract}

\section{INTRODUCTION}

Collective behavior in animal groups is a wide spread phenomenon in biological systems, becoming one of the significant interest topics, have attracted much attention of researchers during the last decade in many different areas of science, including physics, applied mathematics, and engineering. For example, fishes schooling, birds flocking, and insects swarming frequently exhibit complex and coordinated collective behavior and present unrivaled opportunities to link the behavior of individuals with dynamical group-level properties. Fishes schooling can be understood as a self-organized system because they do not need a leader or an external stimulus to avoid splitting up, move cohesively and adopt a common direction. In physics, Vicsek's model $[1,2,8,10]$ have attracted interest 
among researchers, it is a novel type of dynamics in order to investigate phase transition in non-equilibrium systems based on the ferromagnetic spin model, where the velocity and direction of the particles is determined by a simple rule: at each time step, a given particle moves with a constant velocity, a new direction is obtained by the average direction of all the neighborhood particles within a circle of radius $R$ with including a random noise.

Beside VM, the mathematical model is also proposed by Cucker and Smale [3, 6, 12, 13] (CSM) using the equations of classical mechanics. However, these equations expressed in the form of the attraction/repulsion forces which depend on the distance between an individual and its neighbors. Unlike physical model, the equation of motion in CSM is as the separated function of the distance.

In fact, each individual of an animal group prefers to move in the same manner of its neighbors. Of course, it should depend on the position of other ones who are in its viewable area. Thus, in this work, we used the VM to study the effect of view's angle on the phase transition behavior of the fish schools. The model and method are introduced in Sec. II, the critical value of noise $\xi_{c}$ is obtained by the maximum of variance of the average velocity. Section III shows the simulation results and the discussion.

\section{THE MODEL}

The simplest model proposed by Vicsek et al. [1], consists of $N$ individuals continuously move in a plane $(x, y)$ of linear size $L$, the periodic boundary condition is applied to both $x$ and $y$ directions. The individuals are labeled by an index $i$ characterized by their position $\mathbf{r}_{i}=\left(x_{i}, y_{i}\right)$ and velocity $\mathbf{v}_{i}$ of fixed modulus $\left|\mathbf{v}_{i}\right|=v_{0}$.

In this work, we assume the direction of motion of individual $i$ depends on the average velocity of the individuals (including the $i$ th individual) within a circle sector $S(i)$ centered on the individual $i$ with central angle $\phi$ and radius $R$ (see in Fig. 1). For simplicity, we use another notation for the central angle $\varphi=\phi / 2$. In the case $\varphi=\pi$ (i.e. $\phi=2 \pi$ ), the model becomes the original model of Vicsek.

In our simulations, we used the initial conditions: at time $t=0$, the individuals were randomly distributed in the plane with the same absolute velocity $v_{0}$, the direction of motion of all individuals has been randomly chosen in the interval $[0,2 \pi]$.

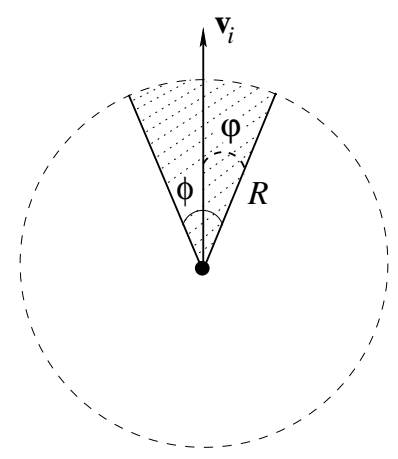

Fig. 1. The angle of view of individual $i$, denote $\varphi=\phi / 2$ is a half of angle of view. 
Base on the definitions in Ref. [1], the position $\mathbf{r}_{i}$ and the direction $\theta_{i}$ of $i$ th individual are updated by the following simple rules

$$
\begin{aligned}
x_{i}(t+\Delta t) & =x_{i}(t)+v_{0} \Delta t \cos \theta_{i}(t), \\
y_{i}(t+\Delta t) & =y_{i}(t)+v_{0} \Delta t \sin \theta_{i}(t), \\
\theta_{i}(t+\Delta t) & =\left\langle\theta_{i}(t)\right\rangle_{S(i)}+\xi,
\end{aligned}
$$

where $\left\langle\theta_{i}(t)\right\rangle_{S(i)}$ is the average direction of the velocity of individuals being within the circle sector $S(i)$. It is given by $\arctan \left(\sum_{j \in S(i)} \sin \theta_{j}(t) / \sum_{j \in S(i)} \cos \theta_{j}(t)\right)$. The last term in Eq. (3) is a random number generated from the uniform distribution on the interval $[-\xi / 2, \xi / 2], \xi$ is so called the "noise" being in a same manner as the temperature parameter in statistical physics.

To study the phase transition, we used the definition of the VM for the absolute value of the average normalized velocity, the order parameter reads

$$
\langle O\rangle \equiv v_{a}=\frac{1}{N v_{0}}\left|\sum_{i=1}^{N} \mathbf{v}_{i}\right| .
$$

The order parameter $\langle O\rangle$ is about zero if the direction of the motion of the individuals is on a random distribution (disordered phase), $\langle O\rangle \simeq 1$ for the parallel motion of the individuals (ordered phase). However, we define a quantity so called the variance of the order parameter $\chi_{O}$ being similar to the definition of susceptibility in the spin models, it can be written as

$$
\chi_{O}=\left\langle O^{2}\right\rangle-\langle O\rangle^{2} .
$$

This quantity is a function of noise $\xi$, then we can obtain the critical value $\xi_{c}$ at the maximum of $\chi_{O}$.

\section{RESULTS AND DISCUSSION}

In all the simulations, we used the interaction radius $R=1$ and time step $\Delta t=1$. The absolute velocity $v_{0}=0.03$ and fixed density of individuals $\rho=N / L^{2} \equiv 1$, where the

size of square $L=\operatorname{int}(\sqrt{N})$ with the total number of individuals $N=40,100,200,300,400$ and 500. Therefore, $N$ is considered as the system size. The angle of view $\phi$ is varied between 0 and $2 \pi$, i.e. $\varphi \in[0, \pi]$.

Figures 2 and 3 show the dependence of $\langle O\rangle$ and $\chi_{O}$ on $\xi$ for several values of view's angle: $\varphi=0.1 \pi, 0.5 \pi$ and $1.0 \pi$, the system size $N=100$. The critical value of noise is determined by the value of $\xi$ at the maximum of $\chi_{O}$ in Fig. $3, \xi_{c}=0.211,0.53$ and 1.75 for $\varphi=0.1 \pi, 0.5 \pi$ and $1.0 \pi$, respectively.

The phase diagram in space $\left(\varphi, \xi_{c}\right)$ is shown in Fig. 4 , where $\varphi=\phi / 2$ (in unit of $\pi$ ). In phase (I), at low noise $\xi<\xi_{c}$, almost of the individuals move in the same direction at a constant speed $v_{0}$ thus the phase is so-called "ordered" phase. Whereas, phase (II) is called "disorder" phase, in which all of individuals have different direction of motion. We see that the phase transition has been separated into two phase at $\varphi=0.5 \pi$, the critical noise $\xi_{c}$ rapidly decreases with decreasing the angle of view $\varphi$. The phase transition is strongly depends on the view's angle $\varphi \geq 0.5 \pi$. For $\varphi<0.5 \pi$, it seems to be independent on the view's angle. 


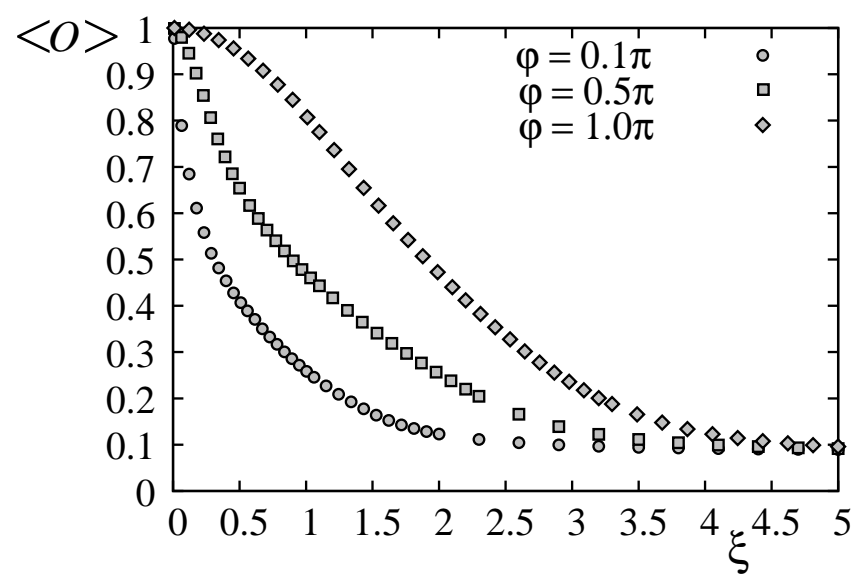

Fig. 2. Order parameter versus noise $\xi$ for several values of $\varphi: 0.1 \pi$ (circles), $0.5 \pi$ (squares) and $1.0 \pi$ (diamonds), with the system size $N=100$.

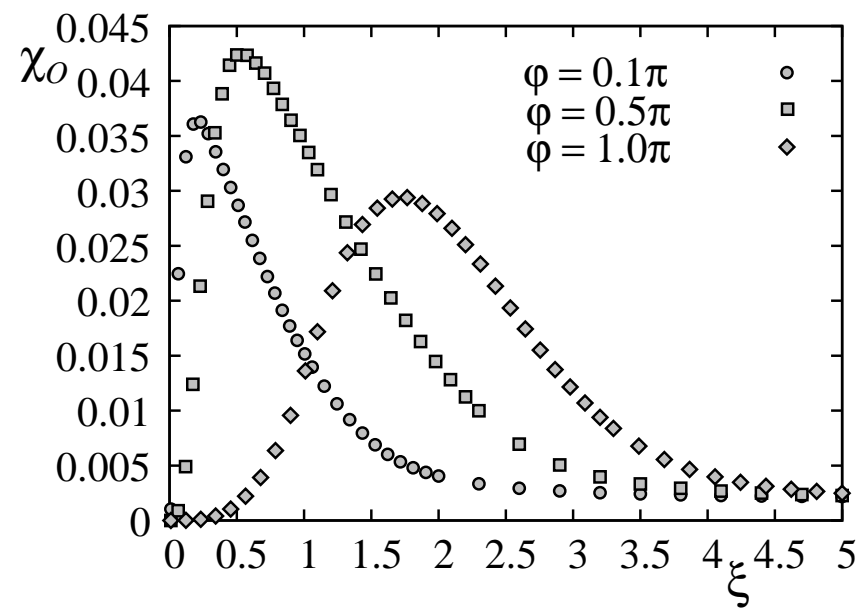

Fig. 3. The variance of the order parameter versus noise $\xi$ for several values of $\varphi: 0.1 \pi$ (circles), $0.5 \pi$ (squares) and $1.0 \pi$ (diamonds), with the system size $N=$ 100.

In summary, based on VM, we studied the effects of view's angle on the phase transition behaviors of fishes schooling system, the phase transition strongly depends on the view's angle of each individual $\varphi$ in range of $(\pi / 2, \pi)$. This range of view's angle is corresponding to the structure and function of eyes of the preys (the viewable area $\phi \in[0,2 \pi]$ or $\varphi>\pi / 2)$, but not of the predators $(\phi \in[0, \pi]$, i.e., $\varphi<\pi / 2)$, so we can conclude that the schooling behavior occurs in the prey species only.

\section{ACKNOWLEDGMENTS}

This research is funded by Vietnam National Foudation for Science and Technology Development (NAFOSTED) under grant number 103.02-2011.55. 


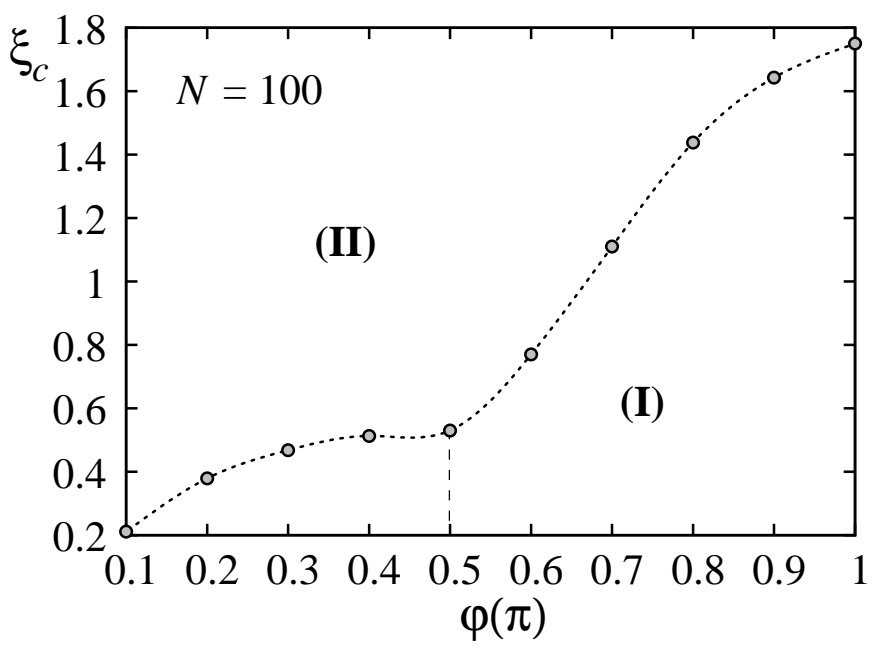

Fig. 4. The phase diagram in space $\left(\varphi, \xi_{c}\right)$ with the system size $N=100$.

\section{REFERENCES}

[1] T. Vicsek, A. Czirok, E. Ben-Jacob, I. Cohen, O. Shochet, Phys. Rev. Lett. 75 (1995) 1226.

[2] A. Czirok, H.E. Stanley, and T. Vicsek, J. Phys. A30 (1996) 137.

[3] F. Cucker and S. Smale, IEEE Trans. Automat. Control 52 (2007) 852-862.

[4] H. Huth, C. Wissel, Ecol. Modelling 75/76 (1994) 135-145.

[5] P. Turchin, Quantitative Analysis of Movement, Sinauer Associated, Inc., Sunderland, 1998.

[6] A. Okubo, Diffusion and ecological problems: mathematical models, Lecture Notes in Biomathematics, Vol. 10. Springer-Verlag, New York, 1980.

[7] E. Klarreich, Science News, 170(22) (2006) 347.

[8] I. D. Couzin, J. Krause, R. James, G. D. Ruxton, N. R. Franks, J. Theor. Biol. 218 (2002) 1-11.

[9] I. D. Couzin, G. D. Krause, N. R. Franks, S. A. Levin, Nature 433 (2005) 513-516.

[10] A. Czirók, M. Vicsek, T. Vicsek, Physica A 264 (1999) 299-304.

[11] Jacques Gautrais, Christian Jost and Guy Theraulaz, Ann. Zool. Fennici 45, (2008) 415-428.

[12] S-Y. Ha and J.-G. Liu, Commun. Math. Sci. 7 (2009) 297-325.

[13] S.-Y. Ha and E. Tadmor, Kinetic and Related Models 1 (2008) 415-435.

[14] Emiliano Cristiani, Paolo Frasca, Benedetto Piccoli. J. Math. Biol. 62, (2011) 569-588. 